\title{
The Psycho-Pedagogical Monitoring Research and the Problems of the Quality of Requirements in the Current Educational System
}

\section{Srbuhi Gevorgyan ${ }^{a}$}

\begin{abstract}
Nowadays, we face many obstacles connected with the high quality education. This sphere forces us to implement new methods and approaches in everyday learning process. The following academic research done on psycho-pedagogical sphere in higher education showed that the enhancement in the educational system is developing slowly because of the lack of research. By all means, it is the right time to follow the objectives and try to develop and suggest the current teaching process using technology that of course will be based on the development of quality control and thesaurus-agency approaches. As mentioned in Bolkan and Goodboy, their research showed that teachers who promote intellectual stimulation empower and enrich students both cognitive and affective getting knowledge and experience process in the classroom. The main objective of current experiment is that it suggests intellectual stimulation which is linked to intrinsic motivation, and that intrinsic motivation has all the advantages to influence students' use of effective studying behaviors. According to Dunlosky, Marsh, Nathan, Willingham and Rawson, there are specific techniques which can influence on the overall process of higher education.
\end{abstract}

Keywords

Psycho-pedagogy, higher education, learning goals, the quality of education

Currently, governmental politics have to solve problems connected with the education system. Those obstacles are mainly connected with the quality of higher education and the professional development of educators. Research done on psycho-pedagogical sphere in higher education showed that the enhancement in the educational system is developing slowly because of the lack of research. It is the right time to follow the objectives and try to develop the current teaching process using technology, which will be based on the development of quality control and thesaurus-agency approaches. Diagnostic technology, based on the methodology of psycho-pedagogical qualimetry and thesaurus approach can be called qualitative, because it is associated with the assessment of the quality of education. Comparing with qualimetry the diagnosis involves qualitative analysis of the empirical data which allows you to put the state of the educational processes. Tracking diagnostic data throughout the test process is known

\footnotetext{
aArmenian State Pedagogical University named after Khachatur Abovyan, Armenia

Correspondent Author:

Srbuhi Gevorgyan, House 15, Komitas 46, Yerevan, Armenia E-mail: gevorgyan_26@mail.ru
} 
as "monitoring”. Psychological monitoring covers all the structural elements and their backbone connection in the psychological system. It plays an essential role in its function and further development. Monitoring the structure of knowledge of students is one of the components of psychological monitoring. It provides continuous monitoring of the training of students that promotes the adoption of timely management decisions and compiling truthful forecasts in the learning process.

Currently in the academic environment, the quality of education is widely discussed, reflecting the desire to upgrade it. This initiates to dig through for approaches to the definition of criteria of quality of education. According to the Bologna reform, quality of higher education is a key condition for the success in higher education. Particular importance is linked to the confluence of quality assurance, accreditation and assessment of educational operation, which is dimensional, complex, and contradictory, challenges not only specific standards, standards, definitions, specifications, or criteria. Perhaps it is impossible to rate educational activities based on the results as positive or negative ones. Sometimes, the results of the educational process fully manifest after a great many years, as during the education process conclusions cannot be accurately recorded. Despite this, they still must be considered in the pedagogical goal setting. For such results, only specific conditions can be created, so that they originate (if it is positive), or, conversely, eliminate the alleged conditions which are known as negative ones. In this case, we can talk about situations in which positive or negative results depend on the hidden or random factors or on specific subjects of the educational process. The results of the educational process differ in the degree of manifestation and the nature of evaluation. Ultimately, the quality of education can be seen not only in terms of knowledge and skills, but also in the motivational sphere of personality and set of stable psychological formations characterizing its culture, social experience.
Therefore, it is necessary to take into account a wide range of quality criteria for education.

\section{CRITERIA FOR IMPROVING HIGHER EDUCATION}

Comparative analysis and logical systematization reveal the following typological groups of criteria, the quality of education at the degree of subject-subject relations: classical, functional, irrational, systematic, and integrated. With this in mind, knowledge, skills, and abilities across the spectrum of its properties and manifestations can be attributed to the group of classic quality criteria. It is essential to mention that conditions for teaching and learning in higher education are changing rapidly, thereby, demand development in teaching practices and the student learning experience. This action was designed to amend plan perspectives, as well as scholarly knowledge arrangement, inside of the educational evolution. As Roxå and Mårtensson (2008) supported, some issues and processes in relation to strategic change in higher education institutions, focus on educational developers and their ability to contribute to the development of teaching and learning. The inclusion of psychological knowledge in the network of on going education, recognition of its worth and importance for individual, apportion of psychological education as necessity, also personality arrangement steps of adulthood enable us to make stronger the need of adult personality achievement to form strong and stable motivation for learning activity during life time. Psycho-pedagogical and socio-psychological analysis of personality structure allow solving this problem and giving its methodological basis. Taking into consideration the fact that each person is independent biologically and socially, supporters of a variety of approaches in psychology demonstrate their own views on the structure of personality. Notwithstanding, there are structural components which have similar content but the variety of integration. Dushka (2014) 
released isolated synthesis of complex substructures orientation such as needs, claims, models, aim, and character. Here we should point out that the devise of identity has been conforming progressively more attention in science education in the past few years, as evidenced in the increased number of different published articles in premier science education journals. According to Scardamalia and Bereiter (2006), the multiple identities that learners and teachers bring with them and further construct and reconstruct in classrooms and out-of-school settings allow them to be, and be accepted as certain types of people. Platonov (1997) explained that social toning (aspect, attitude, and moral uprightness, experience, knowledge, skills, habits) are essential, Rubinstein (1989) pointed to closely interrelated points: the first substantive content, the second is the creation of internal psychological stress. This might denote that according to the requirements, it appears in human beings. Energetic trend may have outside image, and the psychological mechanism, isolated from the viewer "painful” pressure. The aspect is changing, which is provided by accidental conditions and inward deputies; there are also changes in the functional features of personality as the main effect. The specification of the spiritual formation of personality is the accumulation of changes that lead to a qualitative leap in the development, most often occurs hidden without any accidental manifestations. These kinds of changes may be accompanied by deficient actions, accounts, which might be the reason for errors during assessment. According to Platonov (1997), the basis of human activities correspond to some of the components which that particular person experienced. Later on, the author points out the number of basic activities: the value of orientation, cognition, transformation, capabilities to communicate. Apart from the experience, a particular person also is characterized by the level of development of the psycho-functional mechanisms such as development of memory or thinking. And integral typological quality is a product of the individual. Some results of the educational processes can have integrated shade for example competence, extraction, and training. They usually create a group of integrated criteria of quality of education. Future research should be aimed toward developing the technology of psycho-pedagogical diagnostics as a system of component of qualified management education. The variety of tools can be used inside of class setting. Simultaneously, the more evidence-based characteristics your strategy addresses, the more likely the strategy is to reach success. One of the most essential points that we all should pay attention in the education sphere is that.

Currently, there are two main areas in the implementation of quality in higher education: The first is empowering governmental authorities in the operation of qualifying updated education. The second incline is the changeover of these functions in the special social independent organizations: agencies, councils, associations, and corporations. The existence of independent organizations allows you to enter into the system of university management mechanisms, also balances to ensure effective quality control, to preserve academic freedom of universities and the development of self-government. According to Scardamalia and Bereiter (2006), in order to have better developments in the pedagogical sphere or advanced education, knowledge building pedagogy also might be included. This type of theory is mostly based on the premise that authentic creative of fact, it involves students not only developing knowledge-building competencies but also coming to see themselves their work and study. Some psychologists mostly advice to integrate online studying in order to gain better results. In the twenty-first century, that is really essential. To provide students with the education particularly in higher education, they really have to prosper in a globally connected world, we have to find ways to design, fund, construct, and maintain the infrastructure that will make connectivity a reality for every teacher 
and student in each classroom setting. Taking all this in mind, we should be able to integrate online learning in higher education. This type of learning increases the availability of learning experiences for those who cannot or choose not to attend traditional schools, assembling and disseminating instructional content more efficiently, and increases student-instructor ratios while achieving learning outcomes equal to those of traditional classroom instruction (Bakia et al. 2012).

\section{LEARNING TECHNIQUES FOR TEACHERS}

Luckily, cognitive and educational psychologists have been developing and evaluating for years that could be beneficial for students in gaining their learning goals. In this monograph, the authos discusses some learning techniques that offer recommendations about their relative utility. Some of those selected techniques are expected to be relatively easy in order to be adopted by many learners. The described learning techniques will not be a panacea for improving achievement for all students, also, they will benefit for those students who are highly motivated and capable of using them. Notwithstanding, it is susceptible that they will produce meaningful gains in performance in the classroom, on tests, and on many tasks encountered across the life span. So these are some of techniques:

(1) How general are the effects of this technique?

2a. Learning conditions;

2b. Student characteristics;

2c. Materials;

2d. Criterion tasks.

(2) Effects in representative educational contexts

(3) Issues for implementation

(4) Overall assessment (Dunlosky et al. 2013).

The current research showed that there is some intellectual stimulation which has the capability to influence students learning process in higher education. It is really difficult for some time to figure out the specific behaviors students perceive as being transformational in the context of a college classroom setting. Bolkan and Goodboy (2010) conducted an investigation with the aim of creating behavioral measures of transformational leadership for a college context. Thus, results from the research suggest that teachers who promote intellectual stimulation empower and enrich students both cognitive and affective getting knowledge in the classroom. The basic objective of this experiment is that it offers intellectual stimulation which is linked to intrinsic motivation, and that intrinsic motivation has all the advantages to influence students' use of effective studying behaviors. Of interest to this examine, how intrinsic motivation influences three specific studying actions: a deep approach, a conducted approach, and a projecting approach (Duff 2004). The deep, surface, and strategic approaches to studying are associated with germinal research on student learning and reflect students' basic approaches to their school study. Those learners who study using a deep approach look "for meaning in the matter being studied" and relate it to other experiences and points of views Son "rote-learning and memorization in isolation to other ideas” (Duff 2004: 57). On the other hand, learners who study strategically do so in organized and effortful ways, and "optimize success... through effective use of space and time” (Mattick, Dennis, and Bligh 2004: 535). That is really essential how learners approach toward their learning process because research has shown that a deep approach to learning is mostly connected with "higher quality learning outcomes" (Prosser and Trigwell 1999) when analogize to the surface approach, and that general academic interpretation tends to be positively associated with deep and designed approaches. Research by Roxå and Mårtensson (2014) supports that senior teacher's teaching sometimes did not match the teaching quality expected in the unit. They share the idea that academic teaching forms traditions, cultures, and standards that influence individual academics and contribute to the stability in higher 
education often commented upon. Socio-culturally formed collegial contexts at the midlevel of higher education organizations have attracted a great attention from higher education experimenters. It is figured out that development, change, and stability are widely based on standards, habits, and customs in these contexts. The article concludes that the framework presents a promising alternative to state-driven and bureaucratic perspectives as well as to market-inspired perspectives. One formal leader described the efforts to restore a group of teachers where conflicts threatened the standards expected. It was a process stretching over several years and included many types of support. By all means, it is not the absence of problems that signifies these strong micro cultures, but rather the degree of concern and the actions taken to monitor the level of quality aspired to within the unit.

\section{DISCUSSION}

Current academic paper discusses the psycho-pedagogical monitoring research and the main issues connected with the quality of requirements in the current educational system. The most essential frame work is that current higher educational system needs the implantation of new technologies. Teachers should try to use new methods and approaches with students in order to gain better results. However, that is not as easy as seems. Across with the approaches and methods, teachers should try to instrument some basic techniques such as overall assessment, issues for implementation, effects in representative educational contexts (Dunlosky et al. 2013). The overall enhancement of the quality of higher education is developing and growing slowly, so we need so basic changes. It worth to mention that comparative analysis and logical systematization reveal criteria for the quality of education at the degree of subject-subject relations: classical, functional, irrational, systematic, and integrated.

\section{CONCLUSIONS}

This current paper presents the summary of the findings, connected with the psycho-pedagogical sphere in higher education. Our reserach showed that the enhancement in the educational system is developing slowly because of the lack of research. It worths to mention that comparative analysis and logical systematization reveal criteria for the quality of education at the degree of subject-subject. The overall enhancement of the quality of higher education is developing and growing slowly, so we need so basic changes in the particular sphere.

\section{References}

Bakia, M., L. Shear, Y. Toyama, and L. Lasseter. 2012. U.S. Department of Education Office of Educational Technology Center for Technology in Learning SRI International. SRI Publications.

Bolkan, S. and A. K. Goodboy. 2010. Leadership and Intercultural Competence: A Narrative of Transformational Leadership in the 21st Century. Taylor and Freances.

Bolkan, S., A. K. Goodboy, and D. J. Griffin. 2011. Teacher Leadership and Intellectual Stimulation: Improving Students' Approaches to Studying Through Intrinsic Motivation. State University of New York.

Duff, A. 2004. The Role of Cognitive Learning Styles in Accounting Education: Developing Learning Competencies. UK.

Dunlosky, J., E. J. Marsh, M. J. Nathan, D. T. Willingham, and K. A. Rawson. 2013. “Improving Students' Learning With Effective Learning Techniques Promising Directions From Cognitive and Educational Psychology.” Psychological Science in the Public Interest 14(1):4-58.

Dushka, A. L. 2014. "Psychological Assistance to Children With Disorders of the Emotional-Volitional Sphere: Family-Centered Approach.” Fundamental and Applied Researches in Practice of Leading Scientific Schools 2014(6).

Mattick, K., I. Dennis, and J. Bligh. 2004. Approaches to Learning and Studying in Medical Students: Validation of a Revised Inventory and Its Relation to Student Characteristics and Performance. UK: Institute of Clinical Education.

Office of Educational Technology. 2014. About the Professional Learning Strategies Self-Assessment Tool. Retrieved (http://tech.ed.gov/wpcontent/uploads/2014/11/ Section-3-Strategies-Self-Assessment-FINAL.pdf). 
Petorvsky, A. V. and M. T. Yaroshevsky. 1990. Psychology of Words. Moscow: Cultural-Historical Psychology.

Platonov, K. K. 1997. Interesting Psychology. Russia: Rimis Publication.

Prosser, M. and K. Trigwell. 1999. Understanding Learning and Teaching. Buckingham: Open University Press.

Roxå, T. and K. Mårtensson. 2008. "Strategic Educational Development: A National Swedish Initiative to Support Change in Higher Education.” Higher Education Research and Development 27(2):155-168.

- 2014. Higher Education Commons-A Framework for Comparison of Midlevel Units in Higher Education Organizations Academic Development Unit. Faculty of Engineering, Lund University, Lund, Sweden.

Rubinstein, S. L. 1989. Fundamentals of General Psychology. Moscow: Cultural-Historical Psychology.

Scardamalia, M. and C. Bereiter. 2006. "Knowledge Building: Theory, Pedagogy, and Technology.” Pp. 97-118 in Cambridge Handbook of the Learning Sciences, edited by
K. Sawyer. NY: Cambridge University Press.

Seleznyova, N. A. 2004. Quality Assurance and Quality Control in the Specialty of Higher Education. Ufa: All-Russian Conference.

Senashenko, V. S. 2003. Governmental Educational Standards as the Basis of Quality of Education. All-Russian Conference.

Senashenko, V. S. and O. I. Smirnov. 2003. Innovational Processes in Higher Schools. Krasnodar: All-Russian Conference.

\section{Bio}

Srbuhi Gevorgyan, Ph.D. in psychology, professor, Vice Chancellor of Educational and Scientific Affairs, Armenian State Pedagogical University named after Khachatur Abovyan; research fields: psychology of communication, psychology of leadership, and human resource management. 